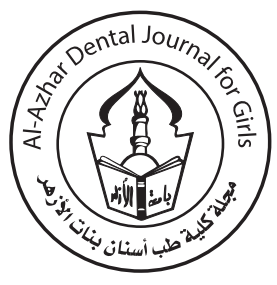

\title{
Antibacterial Effect of Bioactive Composite versus Glass ionomer using Different Storage Media
}

\author{
Walaa M. Hassan ${ }^{1 *}$, Maha A. Niazy $^{2}$, Mayada S. Sultan ${ }^{3}$
}

Codex : 46/20.07

azhardentj@azhar.edu.eg

http://adjg.journals.ekb.eg

DOI: $10.21608 /$ adjg.2020.14224.1188

Restorative Dentistry

(Removable Prosthodontics, Fixed

Prosthodontics, Endodontics, Dental Biomaterials, Operative Dentistry)

\section{KEYWORDS}

Bioactive Composite,

Amalgomer, Glass ionomer.

\begin{abstract}
Purpose: To evaluate the antibacterial effect of Bioactive composite versus Glass ionomer using different storage media. Materials and Methods: Three restorative materials were used in this study (Reliafil Lc, Amalgomer and Conventional Glass Ionomer). 90 Disk- shaped specimens (2.5 mm height and $8 \mathrm{~mm}$ diameter) were prepared from each material. Groups were divided into three subgroups according to storage media (Acids, artificial saliva and de-ionized water). Bacterial strains were polished at $37^{\circ} \mathrm{c}$ for $24 \mathrm{~h}$ in Brain Heart Infusion broth which attained from stock cultures. Disk diffusion agar method was used for antimicrobial liability. The inhibition zone diameter around the specimen were measured at three times by the same operator after $48 \mathrm{hs}$ using electronic digital caliper. Results: This study found that Amalgomer has the highest antibacterial effect in lactic acid. Whereas, Conventional GIC has the highest antibacterial effect in artificial saliva and Bioactive composite in deionized water. Conclusion: This study was concluded that all the restorative material tested had an antibacterial effect in all storage media though Amalgomer seemed to be more prominent in lactic acid.
\end{abstract}

\section{INTRODUCTION}

Dental caries is an infectious microbial disease affecting the hard tooth tissues and results in localized dissolution and destruction of the tooth structures. ${ }^{(1)}$ Streptococcus mutans has a profound effect on the incidence of dental decay in the human population. Streptococcus mutans are able to metabolize sucrose and results in postprandial $\mathrm{PH}$

- Paper extracted from Master thesis titled "Antibacterial Effect of Bioactive Composite versus Glass ionomer using Different Storage Media".

1. Demonstrator of Operative Dentistry, Faculty of Dental Medicine, Nahda University, Egypt

2. Professor of Operative Dentistry, Faculty of Dental Medicine for Girls, Al-Azhar University, Cairo, Egypt.

3. Lecturer of Operative, Faculty of Dental Medicine, Fayoum University, Egypt

* Corresponding author email: walaa.hassan@nub.edu.eg 
drop. ${ }^{(2)}$ Once PH dropped, the hard tooth tissues are demineralized results in dental decay. Dental caries can be prevented by using anti-cariogenic agent. ${ }^{(3)}$

Fluorides play an important role in preventing dental caries as it has an anti-cariogenic property. Fluorides are available in different form and supplements such as mouth rinses, tooth pastes, tablets and restorative materials. ${ }^{(4)}$ Glass ionomers are one of these fluoride-releasing restorative materials. Nowadays, resin composite is the most commonly used restorations. So, researchers try to modify resin composite to be able for fluoride release. ${ }^{(5)}$

Restorative materials with antibacterial functions are very important to inhibit caries, for example glass ionomer was recommended because of their fluoride release ability and adhesive nature to dental hard tissues. Relative lack of strength and low wear resistance are the major limitations of conventional GIC in clinical practice. ${ }^{(6)}$ To overcome this, several modifications in conventional GIC have been done such as resin modified glass ionomer and metal modified GI. ${ }^{(7)}$ Recently, glass ionomers modified by adding ceramic fillers to enhance strength and durability to be like the amalgam restorations without the hazards of mercury. ${ }^{(8)}$

The oral cavity is a complex environment that influence the properties of the restorative materials. Patient habits have a direct effect on oral PH for example, a low-pH sugar containing soft drink causing a high fluoride outflow each time a potential carious site is challenged. ${ }^{\left({ }^{9}\right)}$ It is important to simulate the oral condition with different $\mathrm{PH}$ values to assess fluoride release from dental materials without affecting the physical properties of the material. Glass ionomer cements gradually eroded in the oral environment, although the solubility of glass ionomer under acidic conditions is lower than other cements used in dentistry. ${ }^{(10)}$ Therefore, the null hypothesis of this study, that there is no difference between bioactive composite and glass ionomer regarding the antibacterial effect using different storage media.

\section{MATERIALS AND METHODS}

\section{Materials used in the study:}

In this study, three restorative materials used were Reliafil Lc (Nano hybrid resin composite, Advanced healthcare limited, UK), Amalgomer (Ceramic reinforced GIC, Advanced healthcare limited, UK) and Conventional Glass Ionomer (GC Gold label high strength posterior, Japan).

\section{Sample grouping:}

A total of ninety samples were used in the study. The samples were divided into three groups $(n=30)$ according to material used either Bioactive composite (G1) or Conventional glass ionomer (G2) or Amalgomer (G3). Groups were divided into three subgroups according to storage media (Acids, artificial saliva and de-ionized water).

\section{Preparation of specimens:}

Reliafil LC, thirty disk- shaped specimens of dimensions (2.5 $\mathrm{mm}$ height and $8 \mathrm{~mm}$ diameter) were prepared from each material using split Teflon mold. Sterile split Teflon mold was placed on the sterile glass slap (sterilization were done under ultraviolet UV) and the material was packed into the mold in two increments using sterile gold plated instrument (No5300. Kerr.UK). The first increment was placed into the mold then cured by $3 \mathrm{M}$ ESPE light curing unit (LED curing unit 3M ESPE AG, ESPE Platz, 82229 Seefeld, Germany) for 40 seconds according to manufacturing instructions then the second increment was placed into the mold and celluloid strip was placed to cover the surface. The excess material was removed by steady pressure with another sterile glass slap used on the surface of the mold. Finally, the second increment was cured for 40 seconds according to manufacturing instructions.

For glass ionomer specimens, an equal number of level powder scoops and liquid drops were dispensed on the mixing glass slap; the powder was 
mixed aggressively into the liquid using a sterile cement spatula (metal spatula, ISO 9001:2008, India), until all the powder was incorporated in the mixture. This procedure was done in a maximum of 45 seconds according to manufacturing instructions. The mixed cement was inserted directly by a sterile condenser (metal condenser, ISO 9001:2008, India) into the mold then celluloid strip was placed to cover the surface to assure a flat contact surface, another sterile glass slap was used and the excess material was removed by steady pressure with the same operator. Amalgomer specimens were prepared by the same previously mentioned method except the liquid was distilled water instead of the polyacrylic acid.

\section{Storage of specimens:}

Each subgroup of the three tested materials were stored in the storage media (lactic acid, artificial saliva and deionized water). Each specimen was individually stored in $10 \mathrm{ml}$ of different solutions in graduated sterile plastic containers for $24 \mathrm{hs}$ before subjected to antibacterial test.

\section{Assessment of antibacterial activity:}

Bacterial strains of streptococcus mutans were obtained from stock cultures, cultivated in Brain Heart Infusion broth at $37^{\circ} \mathrm{C}$ for $24 \mathrm{~h}$ and rehydrate the entire pellet with approximately $0.5 \mathrm{ml}$ of broth. Aseptically transfer the entire contents to 5-6 $\mathrm{ml}$ tube of broth. Additional test tubes can be inoculated by transferring $0.5 \mathrm{ml}$ of the primary broth tube to these secondary tubes. Use several drops of the primary broth tube to inoculate broth at $370 \mathrm{c}$ for $48 \mathrm{~h}$. The top $4 \mathrm{~mL}$ of the resulting undisturbed bacterial cultures were transferred to new test tubes and centrifuged for $10 \mathrm{~min}$. The resulting supernatant was discarded and the bacteria (st.mutans) was resuspended in $5 \mathrm{~mL}$ of phosphate buffered saline (PBS) with a $\mathrm{pH}$ of 7.5 and mixed gently for $10 \mathrm{sec}$. The antimicrobial susceptibility profiles were determined by disk diffusion agar method. In each steril- ized Petri dish (20100 mm), a base layer containing $15 \mathrm{~mL}$ of blood agar mixed with $100 \mu \mathrm{l}$ of inoculum was prepared. Each disk- shaped specimen of the tested material was placed in the sterilized Petri dish and incubated at $37^{\circ} \mathrm{C}$ for $48 \mathrm{~h}$ (incubator ZDP-A 050. Shanghai, China). The diameter of zones of inhibition produced around the specimens was measured at three times by the same operator using electronic digital caliper (stainless steel digital caliper (in/mmLCD), China). The diameter of inhibition zones was calculated from the average of these three measurements.

\section{Statistical analysis:}

Standard deviation (SD) and data as mean were calculated for value. Data were used the data distribution, Kolmogorov-Smirnov and Shapiro-Wilk tests. The effect of material and media on mean values were measured usedT-test and ANOVA. Tukey's post-hoc test was used to comparisons when ANOVA test is significant. Significance level was set at $\mathrm{P}=0.05$ and $95 \%$ Confidence interval. Graph Pad Instat (Graph Pad, Inc.) software for wind was performed for statistical analysis.

\section{RESULTS}

Table (1) and (fig.1) represent the significance of the different interactions between three tested materials. With lactic acid; Amalgomer showed the statistically significant highest value $(15.37 \pm 0.87)$ followed by Conventional GIC (13.32 \pm 0.32$)$, while Bioactive composite showed the lowest value (11.69 \pm 0.51$)$.With artificial saliva; Conventional GIC showed the statistically significant highest value $(8.52 \pm 0.19)$ followed by Bioactive composite (7.63 \pm 0.31$)$,while Amalgomer showed the lowest value (6.85 \pm 0.22$)$. With de-ionized water; Bioactive composite showed the statistically significant highest value( $4.50 \pm 0.2)$ followed by Conventional GIC (3.01 \pm 0.11$)$, while Amalgomer showed the lowest value $(2.25 \pm 0.13)$ 
Table (1): Comparison of antibacterial activity results (Mean $\pm S D)$ for all groups with both test conditions.

\begin{tabular}{|c|c|c|c|c|c|}
\hline \multicolumn{2}{|c|}{ Variables } & \multicolumn{3}{c|}{ Test condition } & Statistics \\
\cline { 2 - 6 } & Lactic acid & Artificial saliva & De-ionized water & P value \\
\hline \multirow{3}{*}{$\begin{array}{c}\text { Experimental } \\
\text { groups }\end{array}$} & Amalgomer & $13.32^{\mathrm{B}} \pm 0.32$ & $8.52^{\mathrm{A}} \pm 0.19$ & $3.01^{\mathrm{B}} \pm 0.11$ & $<0.0001^{*}$ \\
\cline { 2 - 6 } & Bioactive composite & $11.69^{\mathrm{C}} \pm 0.51$ & $7.67^{\mathrm{B}} \pm 0.31$ & $4.50^{\mathrm{A}} \pm 0.25$ & $<0.0001^{*}$ \\
\hline \multirow{2}{*}{ Statistics } & P value & $0.001^{*}$ & $0.0005^{*}$ & $<0.0001^{*}$ & $<0.0001^{*}$ \\
\hline
\end{tabular}

Different superscript capital letters (column) indicating significant $(p<0.05) n s$; non-significant $(p>0.05$ *; significant $(p<0.05)$

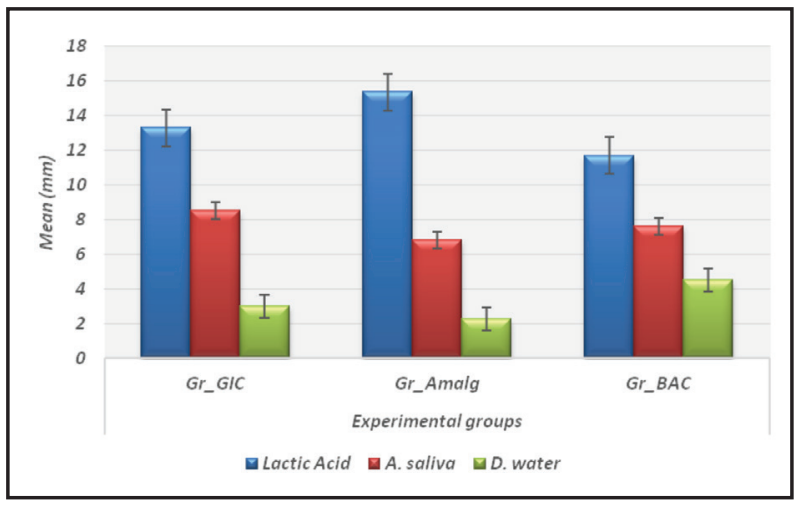

Figure 1: Histogram comparing antibacterial activity of the tested materials.

\section{DISCUSSION}

Prevention of secondary caries around restorative material is dependent on measuring of dental plaque. Though, numerous patients can't care of their oral hygiene perfectly. Antibacterial properties of permanent restorative materials are useful ${ }^{(11)}$. The free fluoride ion stimulates the inhibition of demineralization and improvement of remineralization of the enamel in the oral environment ${ }^{(12)}$. This study was designed to compare antimicrobial effect of different commercially available restorative materials.

Composite restorative materials correspond to one of the many success of modern biomaterials research since they restore biological tissue in both esthetics and function. Composite resin was advanced in atrial to make a composite resin with the fluoride-releasing ability of conventional glassionomer cements. Composite resin and conventional glass ionomer are composed of an equal type of components for example conventional composite resins have large monomer molecules, diluents and particulate inorganic fillers. They contain acidfunctional monomers and small amount of reactive alumina-silicate glass as filler ${ }^{(13)}$.

Glass ionomer cements had been commonly used as a caries preventive aid ${ }^{(14)}$. Fluoride releasing property of glass ionomer cements has been standard in the literature. Fluoride released ions from GIC take part in the demineralization and remineralization phenomena and act directly on the carious process ${ }^{(15)}$.

In this study, the antibacterial activity was designed used the agar diffusion test. This estimation allows bacteria to be screened in a routine, economical and easy way for the detection of resistance ${ }^{(16)}$. Antibacterial effects of restorative materials have been evaluated and the bactericidal effects are often attributed to their low $\mathrm{pH}$ or release of fluoride ${ }^{(17)}$. Fluoride prevents production of bacterial acids and glucans produced by S.mutans. There was a predictable use in testing the antimicrobial activity of restorative materials $^{(18)}$. 
On basis of data from the present study with lactic acid storage medium, Amalgomer showed the statistically significant highest values for the mean diameter of inhibition zone. This may be attributed to the coarse nature of ceramic particles present in Amalgomer that cause an increase in the microporosity of the cement, thus increasing the effective surface area available for elution of fluoride. This finding was in accordance with Bariker and Mandroli (2016) ${ }^{(18)}$ who demonstrated that Amalgomer showed highest antibacterial action against $\mathrm{S}$. mutans and attributed that to ceramic reinforced filler.

Bioactive composite showed the statistically significant lowest value for mean diameter of inhibition zone. This may be due to less amount of fluoride containing glass fillers will be exposed to storage medium after polymerization. This finding was in agreement with Nedeljkovic et al. (2015) ${ }^{(19)}$ who also demonstrated that the bioactive composite exhibited very little antibacterial effect related to the failure to release fluoride.

On basis of data from the present study with artificial saliva storage medium, conventional GIC showed the statistically significant highest value for the mean diameter of inhibition zone. This may be contributing to higher solubility of the cement providing more release of fluoride. These findings were in agreement with study of El-Marakby et al. (2017) ${ }^{(20)}$ which concluded that Conventional GIC showed highest antibacterial action against $\mathrm{S}$. mutans in artificial saliva this may be attributed to their fluoride-leaching capabilities. Also, Tiwari et al. (2016) $^{(21)}$ demonstrated that Conventional GIC has a fast and good antibacterial efficacy attributed to solubility lead to release more fluoride in artificial saliva storage media.

Bioactive composite showed the statistically significant lowest value than conventional glass ionomer for the mean diameter of inhibition zone. This may be due to lose of filler elements by degradation of silane present in interface between matrix and particles which is affecting on components of bioactive composite. This finding was in agreement with Taqa et al. (2019) ${ }^{(22)}$ demonstrated that nano-composite particles lose filler components than micro composite in artificial saliva due to higher exchange substance between artificial saliva and composite (Nano-particles).

Amalgomer showed the statistically significant the lowest value for the mean diameter of inhibition zone. This may be due to low solubility in neutral $\mathrm{pH}$ which has low antibacterial effect. This results were in accordance with Sultan (2019) (23) who found that Amalgomer showed statistically significant the lowest value for the mean diameter of inhibition zone after 48 hours. However, this finding was in disagreement with Hugar et al. (2016) ${ }^{(24)}$ who demonstrated that Amalgomer have high antibacterial effect. This may attribute to the different incubation periods as the antibacterial effect were taken after 1 hour, 1st, 3rd, 5th, and 7th day.

Moreover, the results of the present study showed that Bioactive composite showed the statistically significant the highest value in de-ionized water storage medium for the mean diameter of inhibition zone. This may be attribute to fluoro-boro-alumino silicate glass filler which has antibacterial effect. This finding was in agreement with El zayat et al (2019) ${ }^{(25)}$ who demonstrated that bioactive composite containing (aluminum, boron, silicon, strontium, sodium and fluorine) glass filler act as glass ionomer resulting in inhibition of streptococcus mutans.

Conventional GIC and Amalgomer showed the statistically significant lowest value than Bioactive composite for the mean diameter of inhibition zone. This may be attributed to rapid fall in fluoride release (initial burst) from glass particles as they dissolve in polyalkenolate acid during the setting reaction. This finding was in agreement with Singh et al (2015) ${ }^{(26)}$ who found that rapid fall in fluoride release in first $24 \mathrm{~h}$ only due to initial burst of fluoride release from glass particles as they dissolve in polyalkalonic acid during the setting reaction. 


\section{CONCLUSION}

This study was concluded that all the restorative material tested had an antibacterial effect in all storage media though Amalgomer seemed to be more prominent in lactic acid.

\section{REFERENCES}

1. Pereira C, Cenci MS, Fedorowic Z, Marchesan. Antibacterial agents in composite restorations for the prevention of dental caires. J C D. 2009; 8:201-6.

2. Naik S, Sureshchandra B. Antimicrobial efficacy of glass ionomers, composite resin, liners \&polycarboxylates against selected stock culture microorganisms: an in vitro study. TJPRC. 2012; 24:21-8.

3. Mousavinasab SM, Meyers I. Fluoride release by glass ionomer cements, compomer and giomer. JDRM. 2009; 6:75-81.

4. Silva R, Santos R, Spadaro A. Profile of fluoride release from a nanohybrid composite resin. J D. 2015; 3: 1-4.

5. Buzalaf MA, Passan JP, Honorio HM, Ten Cate JM. Mechanisms of action of fluoride forcaries control. JMOS. $2011 ; 22: 97-114$

6. Ten Cate JM. Contemporary perspective on the use of fluoride products in caries prevention. British Dental Journal. 2013; 214:161-7.

7. Lohbouer U. Dental glass ionomer cements as permenent filling materials. Journal of Dental Materials. 2010;3(1):76-96.

8. Mitra SB, Oxman JD, Falsafi A and Ton TT. Fluoride release and recharge behavior of a nano-filled resin-modified glass ionomer compared with that of other fluoride releasing materials. American Journal of Dentistry. 2011;24:372-8.

9. Hengtrakool C, Kukiattrakoon B, Kedjarune-Leggat U. Effect of naturally acidic agents on microhardness and surface micromorphology of restorative materials. The European Journal of Dentistry. 2011; 5:89-100.

10. Ten Cate JM. Contemporary perspective on the use of fluoride products in caries prevention. British Dental Journal. 2013; 214:161-67.

11. Kampanas N, Antoniadou M. Glass ionomer cements for the restoration of non-carious cervical lesions in the geriatric patient. JFB. 2018; 9:1-9.

12. Madhyastha P, Kotain R, Pai V and Khader A. Fluoride release from glass ionomer cements: effect of temperature, time interval and storage media. Journal of Contemporary Dentistry. 2013; 3:68-73.
13. Porenczuk A, Jankiewicz B, Naurecka M, Bartosewicz B and Sierakowski B. A comparison of the remineralizing potential of dental restorative materials by analyzing their fluoride release profiles. Journal of Advances in Clinical and Experimental Medicine.2019; 28(6):1-9.

14. Tenuta LMA, Cury JA. Fluoride: Its role in dentistry. BOR, 2010; 24:9-17.

15. Vermeersch G, Leloup G, Delmée M, Vreven J Antibacterial activity of glass-ionomer cements, compomers, resin composites: Relationship between acidity and material setting phase. JOR. 2005; 32:368-74.

16. Jorgensen JH, Ferraro MJ. Antimicrobial susceptibility testing: A review of general principles and contemporary practices. JCID. 2009; 49:149-155

17. Zhang K, Wang S, Zhou X,Weir M. Effect of antibacterial dental adhesive on multispecies biofilms formation. JDR 2015; 10:1-8.

18. Bariker R, Mandroli P. An in-vitro evaluation of antibacterial effect of Amalgomer CR and Fuji VII against bacteria causing severe early childhood caries. JISPPD. 2016; 34:183-195.

19. Nedeljkovic I, Teughels W, Munck J, Meerbeek B, Landuyt K. "Is secondary caries with composites a material-based problem?” JDM. 2015; 31:247-7.

20. El-Markby A, Al fawaz S, Alanazi S, Alduaiji $\mathrm{K}$. Evaluation of anti-cariogenic properties among four types of glass ionomer cements. JODH. 2017; 1:1-5.

21. Tiwari S, kenchappa M, Bhayya D, GupTa S, Saxena S, SaTyarTh S, et al. Antibacterial activity and fluoride release of glass ionomer cement, compomer and zirconia reinforced glass-ionomer cement. JCDR. 2016; 10: 90-93.

22. Taqa A, Sulieman R and Al-Sarraf H. Artificial saliva sorption for three different types of dental composite resin (An In Vitro Study). JEDS.2019;18:2339-2344.

23. Sultan M S. Antibacterial effect of aloe Vera and glass ionomer modified by aloe Vera on streptococcus mutans. EDJ. 2019; 65: 1407-1416.

24. Hugar S, Assudani H, Patil V, Shivayogi M, Uppin C and Thakkar P. Comparative evaluation of the antibacterial efficacy of type II glass ionomer cement, type IX glass ionomer cement, and amalgomer ceramic reinforcement by modified "Direct Contact Test": an in vitro study. IJPD. 2016; 9:114-17.

25. El Zayat I, El Banna M and Doaa A.Dintinal microhardness underneath fluoride releasing restorative materials and their associated shear bond strength.EDJ.2019;62:1-12.

26. Singh P, Sawhny A, Singh R, Kumar N, Kumar N and Sajid Z. A comparative evaluation of fluoride releasing capacity of three different restorative materials (conventional glass ionomer, resin modified glass ionomer and poly acid modified composite) in de-ionized water and artificial saliva. IJPCR. 2015; 4:3-6. 